\title{
Quantum Stochastic Differential Equation for Unstable Systems
}

\author{
V.P. Belavkin \\ Mathematics Department, University of Nottingham \\ Nottingham NG7 2RD, United Kingdom \\ P. Staszewski \\ Institute of Mathematics, Pedagogical University of Bydgoszcz \\ 85-072 Bydgoszcz, Poland \\ Received 10 December 1999 \\ Published in: J. Math. Phys. 41 No 11, 2000, 7220-7233.
}

\begin{abstract}
A semi-classical non-Hamiltonian model of a spontaneous collapse of unstable quantum system is given. The time evolution of the system becomes non-Hamiltonian at random instants of transition of pure states to reduced ones, $\eta \mapsto C \eta$, given by a contraction $C$. The counting trajectories are assumed to satisfy the Poisson law. A unitary dilation of the concractive stochastic dynamics is found. In particular, in the limit of frequent detection corresponding to the large number limit we obtain the Itô-Schrödinger stochastic unitary evolution for the pure state of unstable quantum system providing a new stochastic version of the quantum Zeno effect.
\end{abstract}

\section{Introduction and summary}

The decay process is by its nature discontinuous and takes place at random instants of time. Nevertheless, some authors succeeded in describing quantum unstable systems by considering "smoothed" time evolution of unstable systems in the dynamical semigroup approach.

The use of one parameter contracting semigroup in a Hilbert space [1] - 4] for the description of the dynamics of unstable quantum system $\mathcal{S}$ generalizes the law of exponential decay saying that the number of particles in a given state which have not decayed up to $t$ is an exponential function of time; $n(t)=$ $n(0) \exp [-\lambda t], \lambda>0 t \geq 0$. Let $\mathcal{H}$ be a Hilbert space of $\mathcal{S}$, let $\psi(0) \in \mathcal{H}$ denotes an initial (pure) state of $\mathcal{S}$. It is assumed that for any $t \geq 0$ the state of $\mathcal{S}$ is given by formula

$$
\psi(t)=V(t) \psi(0)
$$


where the family $\{V(t), t \geq 0\}$ of bounded operators on $\mathcal{H}$ satisfies the following conditions: (a) $\|V(t)\| \leq 1, t \geq 0$, (b) $V(0)=I$, (c) $V\left(t_{1}+t_{2}\right)=$ $V\left(t_{1}\right) V\left(t_{2}\right), t_{1}, t_{2} \geq 0$, (d) the map $t \mapsto V(t)$ is strongly continuous.

The state (1.1) is normalized to the probability $p(t)=\|\psi(t)\|$ of finding the system undecayed at $t$, moreover $p(t)$ monotonically decreases as the semigroup is contracting.

By virtue of Sz-Nagy theorem [5] there is a unitary dilation of the dynamics $V(t)$ on the Hilbert space $\mathcal{K}=\mathcal{H} \oplus \mathcal{K}$, where $\mathcal{K}$ denotes the Hilbert space of the products of the decay.

Let us assume that the decay of the state of the unstable quantum system $\mathcal{S}$ is represented by completely positive map $\mathcal{I}: T(\mathcal{H}) \rightarrow T(\mathcal{H})$ of the form $[$ ]

$$
\mathcal{I} \rho(t)=C \rho(t) C^{*}, \quad C^{*} C \leq I,
$$

where $I$ is the identity operator in $\mathcal{H}$, the Hilbert space of $\mathcal{S}$. Then the time evolution of the mixed state of the system in question is given by strongly continuous contracting semigroup with the generator of the form [7, 8

$$
\frac{\mathrm{d} \rho^{\lambda}(t)}{\mathrm{d} t}=-\mathrm{i}\left[H, \rho^{\lambda}(t)\right]+\lambda(\mathcal{I}-I) \rho^{\lambda}(t),
$$

where $H$ denotes the hamiltonian of the unstable quantum system $\mathcal{S}$, and $\lambda>0$ is the decay ratio. The mixed state $\rho^{\lambda}(t)$ satisfying the dynamical evolution equation (1.3) is normalized to the survival probability $\operatorname{Tr} \rho^{\lambda}(t)$ for which

$$
\frac{\mathrm{d}}{\mathrm{d} t} \operatorname{Tr} \rho^{\lambda}(t)=\operatorname{Tr}\left[\left(C^{*} C-I\right) \rho^{\lambda}(t)\right] \leq 0 .
$$

In Sect. 2 we give a semi-classical non-Hamiltonian model of spontaneous collapse of an unstable quantum system. The Hamiltonian time-evolution of the system becomes non-Hamiltonian at random instants of transitions $\eta \mapsto C \eta$ of pure states to reduced ones, given by the contraction $C$. It is assumed that the counting trajectories, consisted of instants of occurrences of the collapse, are distributed according to the Poisson law. We find the time-development of the classical state propagator $V_{t}$ in $\mathcal{H}$ in the form of Itô stochastic equation with respect to the classical Poisson process. Consequently, we obtain nonmixing Itô stochastic equations for pure (resp. mixed) states of the unstable quantum system $\mathcal{S}$. It is shown that the averaged density matrix corresponding to the statistical mixture of collapsed states satisfies eq. (1.3). Assuming that each collapse $\eta \mapsto C \eta$ slightly changes the state of $\mathcal{S}\left(I-C=\lambda^{-1} R\right.$ with bounded $R$ satisfying for large $\lambda$ the condition $R^{*} R \leq \lambda\left(R+R^{*}\right)$ ) we find the contracting semigroup equation resulting from the stochastic dynamics in the large number limit $\lambda \rightarrow \infty$.

In Sect. 3 we give the quantum stochastic representation $\widehat{V}_{t}$ of the classical stochastic propagator $V_{t}$ in $\mathcal{H}$ as an operator-valued process in the Hilbert space $\mathcal{H} \otimes \mathcal{F}$, where $\mathcal{F}=F_{+}\left(L^{2}\left(\mathbb{R}_{+}\right)\right)$is the Bose Fock space over the single-particle space of square-integrable complex functions on $\mathbb{R}_{+}$. To this end we employ the generating functional method described in this section. 
As a unitary dilation of a causal contractive cocycle $V_{t}$ in $\mathcal{H}$ cannot in general be obtained from a causal unitary stochastic cocycle $U_{t}$ in the same Hilbert space $\mathcal{H}$, it is impossible to find a Hamiltonian semiclassical dynamics giving the contractive stochastic dynamics of the unstable quantum system as the reduced one. Therefore, we consider the unitary dilation of the contraction $C$ in an extended Hilbert space $\mathcal{H} \otimes \mathbb{C}^{2}$, the latter can be interpreted as the Hilbert space of "quantum meter" detecting the death or life of the unstable particle. The unitary dilation of the contractive stochastic cocycle $V_{t}$, cf. [9], is then realized as a causal unitary cocycle $U_{t}$ in a Hilbert tensor product $\mathcal{H} \otimes \mathcal{F}_{\bullet}$, where $\mathcal{F}_{\bullet}=\mathcal{F}_{+}\left(\mathbb{C}^{2} \otimes L^{2}\left(\mathbb{R}_{+}\right)\right)$, the Bose Fock space over one particle space $\mathbb{C}^{2} \otimes L^{2}\left(\mathbb{R}_{+}\right)$, Sect. 4. We consider two cases of the unitary dilation (4.1) $S$ of $C$ in $\mathcal{H} \otimes \mathbb{C}^{2}$ : (a) with $S$ in the form of Hermitian block-matrix (4.3-4), (b) non-Hermitian unitary block matrix (4.22). In case (a) we find the QSDE for the unitary evolution in $\mathcal{H} \otimes \mathcal{F}$ • with respect to the quantum stochastic Poisson matrix process of intensity $\lambda$. In case (b) we find the limit (as $\lambda \rightarrow \infty$ ) of the unitary evolution using the generating functional method described in Sect. 3. The limiting unitary evolution in $\mathcal{H} \otimes \mathcal{F}$. has the form of the diffusion QSDE with respect to the field momentum process being quantum stochastic representation of the standard Wiener process $w_{t}$ in the Fock space of the representation of the Poisson process. Hence, we obtain (in the representation in which the momentum process is diagonal) that the dilation of the weakly random contractive process with the rate $\lambda \rightarrow \infty$ is described by the Itô-Schrödinger equation for the pure state (in $\mathcal{H}$ ) of the unstable system. The obtained result provides a new stochastic version of the quantum Zeno effect 10, 11, the limiting dynamics becomes reversible as the reductions of decaying amplitude can be compensated by the field fluctuations.

However, while in this paper we do not stress the problem of the Markovian dynamics of a continuously observed (in time) quantum system (the state of which undergoes the collapse), we would like to mention that this important problem of quantum mechanics has been solved in the framework of quantum stochastic calculus, cf. 12, 13, 14, 15, 16, 17] and the literature quoted therein.

\section{A stochastic model for an unstable quantum system}

Now we define a stochastic phenomenological model of spontaneous collapse of an unstable quantum system. It is described as a semiclassical non-Hamiltonian system with a Hilbert space $\mathcal{H}$ of pure quantum states $\eta \in \mathcal{H}$, together with a classical probability space of sequences $\omega=\left\{t_{1}, t_{2}, \ldots\right\} \subset \mathbb{R}_{+}$of the random time instants $t_{1}<t_{2}<\ldots$ of some events (reductions, transitions), which can demolish eventually the quantum system. We shall assume that the sequences $\omega \in \Omega$ are a priori distributed according to the Poisson law, given for each

$t \in \mathbb{R}_{+}$by the "input" probability measure $P_{t}^{\lambda}$ on the measurable subsets of 
finite subsequences $\omega_{t}=\omega \cap[0, t)$ as

$$
P_{t}^{\lambda}(\mathrm{d} \omega)=\lambda^{n} \mathrm{e}^{-\lambda t} \mathrm{~d} t_{1} \mathrm{~d} t_{2} \cdot \ldots \cdot \mathrm{d} t_{n_{t}(\omega)} .
$$

Here $n_{t}(\omega)=\left|\omega_{t}\right|$ is the random number of the events up to time $t, \lambda \geq 0$ is the intensity of the stationary Poisson process $t \mapsto n_{t}$, i.e. the average number of the events per unit of time. The probability of $n$ events, on each interval $[r, r+t)$, is given by the Poissonian distribution

$$
p_{t}^{\lambda}(n)=\int_{r \leq t_{1}<\ldots<t_{n}<t+r} \int_{t}^{\lambda}(\mathrm{d} \omega)=\frac{(\lambda t)^{n}}{n !} \mathrm{e}^{-\lambda t},
$$

independently of $r \in \mathbb{R}_{+}$.

Each event $t \in \omega$ results in an instantaneous change (collapse) $\eta \mapsto C \eta$ of the state of the quantum system, mapping a normalized state $\eta \in \mathcal{H},\|\eta\|^{2}=$ $\langle\eta \mid \eta\rangle=1$ to the reduced state $C \eta$ with the survival probability $\|C \eta\|^{2} \leq 1$. This change satisfies quantum superposition principle i.e. it is described by a linear contraction $C: \mathcal{H} \rightarrow \mathcal{H}, C^{*} C \leq I$. The case $C^{*} C=I$ of isometric $C$ corresponds to a stable (in the positive direction of time) quantum stochastic evolution, with the survival probability one for each state $\eta \in \mathcal{H}$.

If we assume that the quantum system between the reductions is conservative and Hamiltonian, then the nonmixing stochastic evolution $\eta \mapsto \chi_{t}(\omega)$ of the initial quantum normalized states $\eta$ to the pure states $\chi_{t}(\omega) \in \mathcal{H}$ is defined by the measurable maps $\chi_{t}: \Omega \rightarrow \mathcal{H}$ as

$$
\chi_{t}=V_{t} \eta
$$

where

$$
V_{t}(\omega)=\mathrm{e}^{\mathrm{i} H\left(t_{n_{t}(\omega)}-t\right)} C \ldots \mathrm{e}^{\mathrm{i} H\left(t_{1}-t_{2}\right)} C \mathrm{e}^{-\mathrm{i} H t_{1}}=V_{t}\left(t_{1}, \ldots, t_{n}\right) .
$$

Here $\left\{\mathrm{e}^{-\mathrm{i} H t}, t \in \mathbb{R}_{+}\right\}$is a strongly continuous group of unitary operators with a selfadjoint generator $H$ (the hamiltonian of the quantum system in the units $\hbar=1$ ), and for each $t<\infty$ the product (2.4) is finite as $n_{t}(\omega)<\infty$ with probability one. Hence, the stochastic propagator $V_{t}(\omega)$ is well-defined as a contraction in $\mathcal{H}$, giving for each $\omega \in \Omega$ the monotonically decreasing probability of the unstable particle at the time $t$,

$$
\left\|\chi_{t}(\omega)\right\|^{2}=\left\langle V_{t}(\omega) \eta \mid V_{t}(\omega) \eta\right\rangle \leq\left\|\chi_{r}(\omega)\right\|^{2} \leq 1, \quad \forall r \in[0, t) .
$$

Thus, the survival probability $q_{t}(\omega)=\left\|\chi_{t}(\omega)\right\|^{2}$ is obtained as a positive decreasing stochastic process with the initial value $q_{0}(\omega)=1$. Its expectation gives a deterministic monotonically decreasing process of the averaged survival probability

$$
q^{\lambda}(t)=\int q_{t}(\omega) P_{t}^{\lambda}(\mathrm{d} \omega) \leq q^{\lambda}(r) \leq 1, \quad \forall r \in[0, t) .
$$


The stochastic process $q_{t}(\omega)$ defines quantum a posteriori states [6] [3] of the nondemolished quantum system by

$$
\eta_{t}(\omega)=\chi_{t}(\omega) /\left\|\chi_{t}(\omega)\right\|, \quad \forall \omega: q_{t}(\omega) \neq 0,
$$

and the output statistics of the finite sequences $\omega_{t} \subset[0, t)$. The latter is given together with the probability of the survival event of the quantum system at the time $t$ by the output probability measure $Q_{t}^{\lambda}(\mathrm{d} \omega)=q_{t}(\omega) P_{t}^{\lambda}(\mathrm{d} \omega)$, normalized to the probability $q^{\lambda}(t)$. The averaged density matrix

$$
\rho^{\lambda}(t)=\int \rho_{t}(\omega) P_{t}^{\lambda}(\mathrm{d} \omega)=\int \eta_{t}(\omega) \eta_{t}(\omega)^{*} Q_{t}^{\lambda}(\mathrm{d} \omega),
$$

corresponding to the statistical mixture of the collapsed states

$$
\rho_{t}(\omega)=\chi_{t}(\omega) \chi_{t}(\omega)^{*}
$$

by the time $t$ satisfies equation (1.3)

$$
\frac{\mathrm{d} \rho^{\lambda}(t)}{\mathrm{d} t}=-\mathrm{i}\left[H, \rho^{\lambda}(t)\right]+\lambda\left(C \rho^{\lambda}(t) C^{*}-\rho^{\lambda}(t)\right), \quad \rho^{\lambda}(0)=\eta \eta^{*} .
$$

Indeed, this equation can be resolved by the Dyson-von Neumann series [7]

$$
\rho^{\lambda}(t)=\sum_{n=0}^{\infty} \lambda^{n} \int_{0 \leq t_{1}<\ldots<t_{n}<t} \int_{t} V_{t}\left(t_{1}, \ldots, t_{n}\right) \sigma V_{t}\left(t_{1}, \ldots, t_{n}\right)^{*} \mathrm{e}^{-\lambda t} \mathrm{~d} t_{1} \ldots \mathrm{d} t_{n},
$$

which for $\sigma=\eta \eta^{*}$ is the mean value of the stochastic density matrix $\rho_{t}(\omega)$ with respect to the Poisson probability measure (2.2). Thus, the averaged dynamics $\sigma \mapsto \rho^{\lambda}(t)$ for the unstable system is continuous, contractive

$$
\frac{\mathrm{d}}{\mathrm{d} t} \operatorname{Tr} \rho^{\lambda}(t)=\operatorname{Tr}\left[\left(C^{*} C-I\right) \rho^{\lambda}(t)\right] \leq 0,
$$

being normalized to the survival probability $q^{\lambda}(t)=\operatorname{Tr} \rho^{\lambda}(t)$, and mixing.

However, the nonmixing stochastic dynamics

$$
\sigma \mapsto \rho_{t}(\omega)=V_{t}(\omega) \sigma V_{t}(\omega)^{*},
$$

which can be studied in terms of Hilbert space propagators $V_{t}(\omega): \mathcal{H} \rightarrow \mathcal{H}$ is discontinuous and cannot be defined by a differential evolution equation in an ordinary sense. Indeed, the stochastic propagator $V_{t}(\omega)$ is strongly right discontinuous at the points of the collapse $t \in \omega$, but it has strong limits at each $t \in \mathbb{R}$. It is strongly continuous from the left, satisfying the usual Schrödinger equation in terms of the left differentials $\mathrm{d}_{-} V_{t}=V_{t}-V_{t-\mathrm{d} t}=-\mathrm{i} H V_{t}$. However, the Schrödinger equation does not recover the stochastic propagator $V_{t}$ but only its nonstochastic unitary part $\mathrm{e}^{-\mathrm{i} H t}$. 
The proper differential equation for $V_{t}$ can be written as the stochastic equation in Itô sense

$$
\mathrm{d} V_{t}(\omega)+\mathrm{i} H V_{t}(\omega) \mathrm{d} t=(C-I) V_{t}(\omega) \mathrm{d} n_{t}(\omega), \quad V_{0}(\omega)=I .
$$

Here $\mathrm{d} V_{t}$ is forward or symmetric or any other increment of $V_{t}$ but not the backward differential $\mathrm{d}_{-} V_{t}$ for which $\mathrm{d}_{-} n_{t}(\omega)=n_{t}(\omega)-n_{t-\mathrm{d} t}(\omega)=0$ for all $\omega \in \Omega\left(\mathrm{d} n_{t}(\omega)=|\omega \cap[t-\mathrm{d} t, t)|\right.$ is zero as soon as $\mathrm{d} t<t_{n+1}-t_{n}$ for $\left.n=n_{t}(\omega)\right)$.

To be definite, we shall always assume that $\mathrm{d} V_{t}$ (and respectively $\mathrm{d} n_{t}$ ) is the forward differential $V_{t+\mathrm{d} t}-V_{t}$ and $\mathrm{d} n_{t}(\omega)=|\omega \cap[t, t+\mathrm{d} t)|$ is either zero (if $t \notin \omega)$ or one (if $t \in \omega)$ ) for a sufficiently small $\mathrm{d} t\left(\mathrm{~d} t<t_{n+1}-t_{n}\right.$ for $\left.n=n_{t}(\omega)\right)$. Thus the stochastic equation (2.13) coincides with the Schrödinger equation at when there is no collapse, $t \notin \omega$, and $\mathrm{d} V_{t}(\omega)=(C-I) V_{t}(\omega)$ at the points of collapse corresponding to the reduction $V_{t+0}(\omega)=C V_{t}(\omega)$ at $t \in \omega$ and $\mathrm{d} t \rightarrow 0$. One can prove that the stochastic equation (2.13) has only one solution, (2.4). From (2.13) and (2.3) one obtains the stochastic equation for the pure state $\chi_{t}$

$$
\mathrm{d} \chi_{t}(\omega)=-\mathrm{i} H \chi_{t}(\omega) \mathrm{d} t+(C-I) \chi_{t}(\omega) \mathrm{d} n_{t}(\omega), \quad \chi_{0}(\omega)=\eta .
$$

The stochastic density matrix (2.9) can also be obtained by iterations as the unique solution to the stochastic differential equation

$$
\mathrm{d} \rho_{t}=-\mathrm{i}\left[H, \rho_{t}\right] \mathrm{d} t+\left(C \rho_{t} C^{*}-\rho_{t}\right) \mathrm{d} n_{t}, \quad \rho_{0}(\omega)=\sigma .
$$

Note, that this equation coinciding with the von Neumann equation, $\mathrm{d} \rho_{t} / \mathrm{d} t=$ $-\mathrm{i}\left[H, \rho_{t}\right]$ at $t \notin \omega$ and with $\mathrm{d} \rho_{t}=C \rho_{t} C^{*}-\rho_{t}$ at the points of the collapse $\rho_{t+0}(\omega)=C \rho_{t}(\omega) C^{*}, t \in \omega$, can be derived from the stochastic equation (2.14). Indeed, by virtue of the Itô differentiation formula applied to the product $\chi_{t} \chi_{t}^{*}$ :

$$
\mathrm{d}\left(\chi_{t} \chi_{t}^{*}\right)=\mathrm{d} \chi_{t} \cdot \chi_{t}^{*}+\chi_{t} \cdot \mathrm{d} \chi_{t}^{*}+\mathrm{d} \chi_{t} \cdot \mathrm{d} \chi_{t}^{*}
$$

and the Itô multiplication table

$$
(\mathrm{d} t)^{2}=0, \quad\left(\mathrm{~d} n_{t}\right)^{2}=\mathrm{d} n_{t}, \quad \mathrm{~d} n_{t} \mathrm{~d} t=0=\mathrm{d} t \mathrm{~d} n_{t}
$$

one easily obtains (2.15). Then, the averaged mixing equation for $\rho_{t}^{\lambda}$ is obtained from (2.15) by formal replacement $\mathrm{d} n_{t}$ with $\lambda \mathrm{d} t$ corresponding to the averaged number $n_{t}^{\lambda}=\lambda t$ for the Poisson process with the intensity $\lambda$.

The strongly continuous nonmixing evolution

$$
\rho(t)=\mathrm{e}^{-K t} \sigma \mathrm{e}^{-K^{*} t},
$$

with

$$
K=\mathrm{i} H+\lambda(I-C)
$$

corresponding to equation (1.3) follows from (2.15) in the large number limit $\lambda \rightarrow \infty$ of the stochastic evolution under the condition that each collapse $\eta \mapsto$ $C \eta$ only slightly changes the state of the unstable system such that $\eta-C \eta$ is inversely proportional to $\lambda$. Indeed, substituting in equations (2.13), (2.15) 
$I-C$ by $\lambda^{-1} R$, where $R$ satisfies the condition $R^{*} R \leq \lambda\left(R+R^{*}\right)$ for large $\lambda$, we obtain

$$
\mathrm{d} V_{t}(\omega)+\left(R \lambda^{-1} \mathrm{~d} n_{t}(\omega)+\mathrm{i} H \mathrm{~d} t\right) V_{t}(\omega)=0 .
$$

As in the large number limit $\lambda^{-1} n_{t}(\omega)$ converges to $t$ with probability one, this dynamics becomes nonstochastic, satisfying the ordinary differential equation

$$
\frac{\mathrm{d}}{\mathrm{d} t} V(t)+K V(t)=0, \quad V(0)=I,
$$

for $V(t)=\lim _{\lambda \rightarrow \infty} V_{t}=V_{t}^{0}$ with $K=\mathrm{i} H+R$. It has a unique strongly continuous solution $\mathrm{e}^{-K t}$, which is a semigroup of contractions as $K+K^{*}=R+R^{*} \geq 0$. The corresponding nonmixing equation

$$
\frac{\mathrm{d}}{\mathrm{d} t} \rho(t)+K \rho(t)+\rho(t) K^{*}=0
$$

for nonstochastic density matrix $\rho(t)=V(t) \sigma V^{*}(t)$ can be obtained in the limit $\lambda \rightarrow \infty$ from (2.10), or directly from Itô equation (2.15) with $C=I-\lambda^{-1} R$. This is not surprising as the large number limit coincides with its average, thus becoming nonmixing in this limit.

\section{A generating functional method and quantum stochastic representation}

A very convenient method of treating stochastic equations is based on studying the corresponding generating functional equations. The generating functional for a causal stochastic process $\chi_{t}(\omega)$ obtained by solving a stochastic equation with respect to the Poisson process of the intensity $\lambda$ is defined as the averaged product $\chi_{t}^{f}=\chi_{t} \varepsilon_{t}^{f}$,

$$
\breve{\chi}_{t}(f)=\left\langle\chi_{t}^{f}\right\rangle:=\int \chi_{t}(\omega) \varepsilon_{t}^{f}(\omega) P_{t}^{\lambda}(\mathrm{d} \omega),
$$

where $\varepsilon_{t}^{f}(\omega)$ is the stochastic exponent for the martingale process $m_{t}=n_{t}-\lambda t$, satisfying the stochastic equation

$$
\lambda^{1 / 2} \mathrm{~d} \varepsilon_{t}^{f}(\omega)=f(t) \varepsilon_{t}^{f}(\omega) \mathrm{d} m_{t}(\omega), \quad \varepsilon_{0}^{f}(\omega)=1 .
$$

Here $f(t)$ is a nonstochastic complex locally integrable test function such that $\left|1+\lambda^{-1 / 2} f(t)\right| \leq 1$ for all $t$. The solution to this stochastic equation can be written as

$$
\varepsilon_{t}^{f}(\omega)=\exp \left[-\lambda^{1 / 2} \int_{0}^{t} f(r) \mathrm{d} r\right] \prod_{r \in \omega_{t}}\left(1+\lambda^{-1 / 2} f(r)\right),
$$


where $\omega_{t}=\omega \cap[0, t)$. The inverse transform $\breve{\chi}_{t} \mapsto \chi_{t}$ can be written in terms of the series of iterated stochastic integrals

$$
\int \lambda^{-|\tau| / 2} \varphi(\tau) \mathrm{d} m_{\tau}:=\sum_{n=0}^{\infty} \lambda^{-n / 2} \int_{0 \leq r_{1}<\ldots<r_{n}<\infty} \int_{1} \varphi\left(r_{1}, \ldots, r_{n}\right) \mathrm{d} m_{r_{1}} \ldots \mathrm{d} m_{r_{n}},
$$

as $\chi_{t}(\omega)=\int \lambda^{-|\tau| / 2} \widetilde{\chi}_{t}(\tau) \mathrm{d} m_{\tau}$, where $\tilde{\chi}_{t}\left(r_{1}, \ldots, r_{n}\right)$ are the functional derivatives of $\breve{\chi}_{t}(f)$ with respect to $f\left(r_{1}\right), f\left(r_{2}\right), \ldots, f\left(r_{n}\right)$;

$$
\tilde{\chi}_{t}\left(r_{1}, \ldots, r_{n}\right)=\delta^{n} \breve{\chi}_{t}(f) /\left.\delta f\left(r_{1}\right) \ldots \delta f\left(r_{n}\right)\right|_{f=0} .
$$

In particular, the stochastic exponent $\varepsilon_{t}^{g}$ has the exponential generating functional

$$
\breve{\varepsilon}_{t}^{g}(f)=\int \varepsilon_{t}^{f}(\omega) \varepsilon_{t}^{g}(\omega) P_{t}^{\lambda}(\mathrm{d} \omega)=\mathrm{e}^{\int_{0}^{t} g(r) f(r) \mathrm{d} r}
$$

such that $\breve{\varepsilon}_{t}^{g}(f)=\breve{\varepsilon}_{t}^{f}(g)$. Indeed, it follows from the multiplication formula for stochastic exponents,

$$
\varepsilon_{t}^{f} \varepsilon_{t}^{g}=\varepsilon_{t}^{f \dot{+} g} \mathrm{e}_{0}^{t} g(r) f(r) \mathrm{d} r,
$$

where

$$
f \dot{+} g=f+\lambda^{-1 / 2} f g+g
$$

and $\left\langle\varepsilon_{t}^{f \dot{+} g}\right\rangle=1$ as it is easily seen in the explicit representation (3.3). Note that $\varepsilon_{t}^{g}$ can be written in the form of the multiple integral (3.4) as $\varepsilon_{t}^{g}=\varepsilon^{g_{t}}$,

$$
\varepsilon^{g}=\sum_{n=0}^{\infty} \lambda^{-n / 2} \int_{0 \leq r_{1}<\ldots<r_{n}<\infty} \int_{1} g\left(r_{1}\right) \ldots g\left(r_{n}\right) \mathrm{d} m_{r_{1}} \ldots \mathrm{d} m_{r_{n}},
$$

where $g_{t}(r)=g(r), r<t$ and $g_{t}(r)=0, r \geq t$. This follows from $\breve{\varepsilon}_{t}^{g}(f)=\breve{\varepsilon}^{g_{t}}(f)$, where $\breve{\varepsilon}^{g}(f)=\exp \left\{\int_{0}^{\infty} g(r) f(r) \mathrm{d} r\right\}$ corresponds to the kernel

$$
\widetilde{\varepsilon}^{g}\left(r_{1}, \ldots, r_{n}\right)=g\left(r_{1}\right) \ldots g\left(r_{n}\right) .
$$

Note, that the Hilbert space $L_{P}^{2}(\Omega)$ of complex random functions $\chi(\omega)$ with $\int_{\Omega}|\chi(\omega)|^{2} P(\mathrm{~d} \omega)<\infty$ is isomorphic to the Fock space of their transforms $\tilde{\chi}$ with respect to the scalar product

$$
(\varphi \mid \widetilde{\chi})=\sum_{n=0}^{\infty} \int_{0 \leq r_{1}<\ldots<r_{n}<\infty} \int_{\varphi} \bar{\varphi}\left(r_{1}, \ldots, r_{n}\right) \widetilde{\chi}\left(r_{1}, \ldots, r_{n}\right) \mathrm{d} r_{1} \ldots \mathrm{d} r_{n} .
$$

Thus, the generating functional (3.1) can be written in terms of the scalar product (3.11) as follows

$$
\breve{\chi}_{t}(\bar{g})=\left(\widetilde{\varepsilon}_{t}^{g} \mid \widetilde{\chi}_{t}\right)=\int_{\tau \subset[t, 0)} \overline{\widetilde{\varepsilon}_{t}^{g}}(\tau) \widetilde{\chi}_{t}(\tau) \mathrm{d} \tau
$$


for the tilde transform (3.10) of (3.9) and $\tilde{\chi}_{t}$.

Let us now obtain a differential equation for the generating functional $\breve{\chi}_{t}$ of the stochastic process $\chi_{t}$, satisfying the equation (2.14). By differentiating the pointwise product $\chi_{t}^{f}(\omega)=\chi_{t}(\omega) \varepsilon_{t}^{f}(\omega)$ we obtain the stochastic equation

$$
\mathrm{d} \chi_{t}^{f}+\left(\lambda^{1 / 2} f(t)+\mathrm{i} H\right) \chi_{t}^{f} \mathrm{~d} t=\left(C\left(1+\lambda^{-1 / 2} f(t)\right)-I\right) \chi_{t}^{f} \mathrm{~d} n_{t},
$$

from (2.14) and (3.2) by applying the Itô formula

$$
\begin{aligned}
\mathrm{d}\left(\chi_{t} \varepsilon_{t}^{f}\right) & =\mathrm{d} \chi_{t} \cdot \varepsilon_{t}^{f}+\chi_{t} \cdot \mathrm{d} \varepsilon_{t}^{f}+\mathrm{d} \chi_{t} \cdot \mathrm{d} \varepsilon_{t}^{f} \\
& =\left[(C-I) \mathrm{d} n_{t}-\mathrm{i} H \mathrm{~d} t+\lambda^{-1 / 2} f(t) \mathrm{d} m_{t}+(C-I) \lambda^{-1 / 2} f(t) \mathrm{d} n_{t}\right] \chi_{t}^{f} .
\end{aligned}
$$

Thus, the generating functional $\breve{\chi}_{t}(f)=\left\langle\chi_{t}^{f}\right\rangle$ satisfies the ordinary differential equation

$$
\frac{\mathrm{d}}{\mathrm{d} t} \breve{\chi}_{t}+\mathrm{i} H \breve{\chi}_{t}=(C-I)\left(\lambda^{-1 / 2} f(t)+1\right) \lambda \breve{\chi}_{t}
$$

with the initial condition $\breve{\chi}_{0}(f)=\eta$ for all $f$. The increment $\mathrm{d} n_{t}$ is replaced in (3.15) by its average $\left\langle\mathrm{d} n_{t}\right\rangle=\lambda \mathrm{d} t$ because it does not depend on $\chi_{t}$. The solution to this equation can be written in terms of time ordered exponents $\breve{\chi}_{t}=\overleftarrow{\exp }\left[-\int_{0}^{t} K^{\lambda}(r) \mathrm{d} r\right] \eta$ as follows

$$
\breve{\chi}_{t}=\sum_{n=0}^{\infty}(-1)^{n} \int_{0 \leq r_{1}<\ldots<r_{n}<t} \int^{\lambda} K^{\lambda}\left(r_{n}\right) \ldots K^{\lambda}\left(r_{1}\right) \eta \mathrm{d} r_{1} \ldots \mathrm{d} r_{n},
$$

where

$$
K^{\lambda}(r)=\lambda(I-C)\left(I+\lambda^{-1 / 2} f(r)\right)+\mathrm{i} H .
$$

Thus, the tilde transform $\widetilde{\chi}_{t}$ of the stochastic function $\chi_{t}$ is given by

$$
\tilde{\chi}_{t}\left(r_{1}, \ldots, r_{n}\right)=\mathrm{e}^{\left(r_{n}-r\right) K}(C-I) \ldots \mathrm{e}^{\left(r_{1}-r_{2}\right) K}(C-I) \mathrm{e}^{-r_{1} K} \eta,
$$

where $K$ is given by formula (2.19).

It is particularly simple to obtain the large number limit in terms of the generating functional, one has $\breve{\chi}_{t}(f) \rightarrow \mathrm{e}^{-K t} \eta$ as $\lambda \rightarrow \infty$ under the condition $\lambda(I-C) \rightarrow R$, since obviously $K^{\lambda}(t) \rightarrow R+\mathrm{i} H$.

It is well known [18] that the classical stochastic Poisson process $n_{t}(\omega)$ has a quantum field representation $N_{t}=\widehat{n}_{t}$ in the Bosonic Fock space $\mathcal{F}$ over the single quantum space $L^{2}\left(\mathbb{R}_{+}\right)$of square-integrable complex functions on $\mathbb{R}_{+}$in terms of the basic quantum stochastic processes of number $\Lambda_{t}$, creation $A_{t}^{*}$, and annihilation $A_{t}$ on the interval $[0, t)$. Let us also find the corresponding quantum stochastic representation for the stochastic process $\chi_{t}$ satisfying the equation (2.14).

Realizing $\mathcal{F}$ as the space of square-integrable summable functions $\varphi$ of the finite, ordered sequences $\tau=\left(r_{1}, \ldots, r_{n}\right), r_{1}<\ldots<r_{n}$,

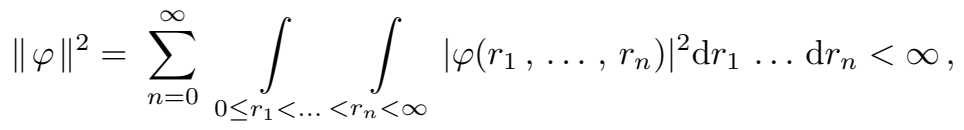


we can represent the canonical operator processes $A_{t}, A_{t}^{*}, \Lambda_{t}$ as

$$
A_{t} \varphi(\tau)=\int_{0}^{t} \dot{\varphi}(\tau, r) \mathrm{d} r, \quad A_{t}^{*} \varphi(\tau)=\sum_{r \in \tau} \varphi(\tau \backslash r), \quad \Lambda_{t} \varphi(\tau)=|\tau| \varphi(\tau)
$$

Here $n_{t}=\left|\tau_{t}\right|$ is the length of a subsequence $t_{1}, \ldots, t_{n_{t}}<t$ of the sequence $\tau$ with $t_{n_{t+1}} \geq t, \tau \backslash r$ is the subsequence without an element $r \in \tau$, and $\dot{\varphi}(\tau, r)=\varphi(\tau \sqcup r)$, where $\tau \sqcup r$ is the ordered sequence with an additional element $r \notin \tau$.

Now, one can define the operator-valued representation $M_{t}=\widehat{m}_{t}$ of the stochastic processes $m_{t}=n_{t}-\lambda t$ by the sum

$$
M_{t}=\Lambda_{t}+\sqrt{\lambda}\left(A_{t}+A_{t}^{*}\right)
$$

Any regular quantum stochastic process $X_{t}$ which is adapted with respect to the family of commuting selfadjoint operators $\left\{M_{t}, t \in \mathbb{R}_{+}\right\}$in $\mathcal{F}$ is given by the series of iterated integrals

$$
X_{t}:=\sum_{n=0}^{\infty} \lambda^{-n / 2} \int_{0 \leq r_{1}<\ldots<r_{n}<t} \int_{\chi} \tilde{\chi}\left(r_{1}, \ldots, r_{n}\right) \mathrm{d} M_{r_{1}} \ldots \mathrm{d} M_{r_{n}} .
$$

The map $\tilde{\chi}_{t} \mapsto X_{t}$ is one-to-one because the kernel $\widetilde{\chi}_{t}$ in (3.22) is uniquely defined as the image $\breve{X}_{t}:=X_{t} \varphi_{0}$ of $X_{t}=\int \tilde{\chi}(\tau) \mathrm{d} M_{\tau}$ on the vacuum state $\varphi_{0}(\tau)=\delta_{0}^{|\tau|}\left(\varphi_{0}\right.$ is equal to zero if $n=|\tau| \neq 0$ and is equal to one if $\left.\tau=\emptyset\right)$. If the kernel $\widehat{\chi}_{t}$ is given by the functional derivatives (3.5) of the functional $\breve{\chi}_{t}$, (3.22) can be formally written as the normally ordered causal expression $X_{t}=: \breve{\chi}_{t}\left(\lambda^{-1} \dot{M}\right)$ : of the quantum field $\widehat{f}=\lambda^{-1} \dot{M}_{t}$, where $\dot{M}$ is the generalized time derivative of (3.21). The composition of the map $\breve{\chi}_{t} \mapsto X_{t}$ with the map $\chi_{t} \mapsto \breve{\chi}_{t}$ in (3.1) defines an operator representation $\chi_{t} \mapsto X_{t}$ called the quantum stochastic representation of the process $\chi_{t}$. In particular, the Wick exponent

$$
W_{t}^{g}=\int_{\tau \subset[0, t)} \prod_{r \in \tau}\left(g(r) / \lambda^{1 / 2}\right) \mathrm{d} M_{\tau}=\widehat{\varepsilon}_{t}^{g}
$$

defined as the unique solution to the operator differential equation

$$
\lambda^{1 / 2} \mathrm{~d} W_{t}^{f}=f(t) W_{t}^{f} \mathrm{~d} M_{t}, \quad W_{0}^{f}=\widehat{1},
$$

in terms of forward differentials $\mathrm{d} M_{t}=M_{t+\mathrm{d} t}-M_{t}$, is the quantum stochastic integral representation (3.3) of the solution to the stochastic differential equation (3.2) with the tilde transform $\widetilde{\varepsilon}_{t}^{f}(\tau)=W_{t}^{f} \varphi_{0}$. It has the operator multiplication

$$
W_{t}^{f} W_{t}^{g}=W_{t}^{f \dot{+} g} \exp \left\{\int_{0}^{t} f(r) g(r) \mathrm{d} r\right\}
$$

representing the stochastic multiplication (3.6), and can be formally written as the normally ordered exponent of $\lambda^{-1 / 2} \int_{0}^{t} g(r) \mathrm{d} M(r)$ having the Wick symbol 
(3.6). From this it follows that

$$
\left(W_{t}^{g} \varphi_{0} \mid X_{t} \varphi_{0}\right)=\left(W_{t}^{g} \varphi_{0} \mid X_{t} \varphi_{0}\right)=\left\langle\widetilde{\varepsilon}_{t}^{g} \mid \widetilde{\chi}_{t}\right\rangle=\breve{\chi}_{t}(\bar{g}),
$$

where $\breve{\chi}_{t}$ is the generating functional of a causal stochastic process with the tilde transform $\tilde{\chi}_{t}$. Thus the generating functional $\left(X_{t}^{f}\right)=\left(\varphi_{0} \mid X_{t}^{f} \varphi_{0}\right)$, defined for the operator integral $X_{t}=\widehat{\chi}_{t}$ as the vacuum expectation of the commuting products $X_{t}^{f}=X_{t} W_{t}^{f}$, coincides with the generating functional for the classical stochastic process (3.4). This also proves the statistical equivalence of the classical process $\chi_{t}$ and the quantum process $X_{t}=\widehat{\chi}_{t}$, having the kernel $\widetilde{\chi}_{t}=\breve{X}_{t}$ as the tilde transform of $\chi_{t}$.

Now, we can define a quantum stochastic representation $\widehat{V}_{t}$ of classical stochastic propagator $V_{t}(\omega)$ in $\mathcal{H}$ as an operator-valued process acting in the Hilbert product $\mathcal{H} \otimes \mathcal{F}$ by the quantum stochastic differential equation

$$
\mathrm{d} \widehat{V}_{t}+\mathrm{i} H \widehat{V}_{t} \mathrm{~d} t=(C-I) \widehat{V}_{t} \mathrm{~d} N_{t}, \quad \widehat{V}_{0}=I \otimes \widehat{1},
$$

where the operators $H$ and $C$ act in $\mathcal{H} \otimes \mathcal{F}$ as $H \otimes \widehat{1}$ and $C \otimes \widehat{1}$, and $N_{t}=M_{t}+\lambda t \widehat{1}$.

The tilde transform $\widetilde{V}_{t}(\tau)$ of $V_{t}(\omega)$ is the kernel for the process $\widehat{V}_{t}$, and the generating functional $\breve{V}_{t}$ coincides with the vacuum conditional expectation $\breve{V}_{t}(f)=F_{0}^{*} \widehat{V}_{t}^{f} F_{0}$ for $\widehat{V}_{t}^{f}=\widehat{V}_{t}\left(I \otimes W_{t}^{f}\right)$, given by the isometry $F_{0} \eta=\eta \otimes \varphi_{0}$ of the Hilbert space $\mathcal{H}$ to $\mathcal{H} \otimes \mathcal{F}$. It satisfies the ordinary differential equation (3.15) for each $\eta \in \mathcal{H}$ as $\breve{\chi}_{t}=\breve{V}_{t} \eta$

$$
\frac{\mathrm{d}}{\mathrm{d} t} \breve{V}_{t}+\mathrm{i} H \breve{V}_{t}=(C-I)\left(\lambda^{-1 / 2} f(t)+1\right) \lambda \breve{V}_{t}
$$

with the initial condition $\breve{V}_{0}(f)=I$ for all $f$.

\section{A unitary dilation of the contractive stochas- tic dynamics}

A unitary dilation of a causal contractive cocycle $V_{t}(\omega)$ in $\mathcal{H}$ (9], cf. also [19]) cannot in general be obtained from a causal unitary stochastic cocycle $U_{t}\left(\omega^{0}, \omega^{1}\right)$ in the same Hilbert space $\mathcal{H}$ by fixing $\omega^{0}=\omega$ and averaging over additional degrees of randomness $\omega^{1} \in \Omega^{1}$. (This is not correct unless like in our paper only classical randomness is considered.) Even a single contraction $C$ might not be represented as a classical mean $\sum_{k} S_{k} \lambda_{k}$ of a random unitaries $S_{k}$ with some probabilities $\lambda_{k} \geq 0, \sum_{k} \lambda_{k}=1$. This makes it impossible to find a Hamiltonian semiclassical dynamics giving the contractive stochastic dynamics of an unstable quantum system as a result of a reduced description. However, it can be obtained from a unitary operator $S$ in an extended Hilbert space $\mathcal{H} \otimes \mathcal{K}$ as a block-matrix element

$$
C=(I \otimes e)^{*} S\left(I \otimes e_{0}\right), \quad\|e\|=1=\left\|e_{0}\right\| .
$$


Such a dilation describes the contraction $C$ by the probability amplitudes

$$
\left\langle\eta \otimes e \mid S\left(\eta_{0} \otimes e_{0}\right)\right\rangle=\left\langle\eta \mid C \eta_{0}\right\rangle
$$

of the unitary transitions $\eta_{0} \otimes e_{0} \rightarrow \eta \otimes e$ in $\mathcal{H} \otimes \mathcal{K}$, given by the fixed unital vectors $e_{0}, e \in \mathcal{K}$, as the probability amplitudes of the contractive transitions $\eta_{0} \rightarrow \eta$. The unitary dilation $S$ of the contraction $C$ can always be built in the Hilbert space $\mathcal{H} \oplus \mathcal{H}$ with the help of two dimensional space $\mathcal{K}=\mathbb{C}^{2}$ by realizing $S$ as a Hermitian block-matrix

$$
S=\left(\begin{array}{cc}
S_{0}^{0} & S_{1}^{0} \\
S_{0}^{1} & S_{1}^{1}
\end{array}\right), \quad S_{0}^{0 *}=S_{0}^{0}, \quad S_{0}^{1 *}=S_{1}^{0}, \quad S_{1}^{0 *}=S_{0}^{1}, \quad S_{1}^{1 *}=S_{1}^{1}
$$

with the transition elements $S_{0}^{1}=C, S_{1}^{0}=C^{*}$ and

$$
S_{0}^{0}=-\left(I-C^{*} C\right)^{1 / 2}, \quad S_{1}^{1}=\left(I-C C^{*}\right)^{1 / 2} .
$$

The unitarity $S^{-1}=S^{*}$ of (4.2) simply follows from $C S_{0}^{0}+S_{1}^{1} C=0, S_{0}^{0} C^{*}+$ $C^{*} S_{1}^{1}=0$. We can interpret the unit basic vectors $e_{0}, e_{1} \in \mathcal{K}$ as the eigenstates of a quantum meter detecting the death or life of the unstable particle, correspondingly. In the case $C C^{*}=I$ of coisometric $C$ the unitary operator $S$ describes a transition of the input particle-meter states $\eta \otimes e_{0}, e_{0}=\left(\delta_{0}^{k}\right)$ to a superposition of the alive states $\eta^{1}=C \eta$, corresponding to the vector $e_{1}=\left(\delta_{1}^{k}\right)$, and the dead states $\eta_{0}=-\eta^{\perp}$, where $\eta^{\perp}=\eta-\eta^{\prime}$ is the orthogonal projection to $\eta^{\prime}=C^{*} C \eta$. But the alive states $\eta \otimes e_{1}$ transit only to the states $C^{*} \eta \otimes e_{0}$ corresponding to the exiting of the particle from the detector. Thus, for realization (4.2) with the input "vacuum" vector $e_{0}$, the output vector $e$ in (4.1) is the vector $e_{1}$ corresponding to the detection of the unstable particle. The described unitary dilation of the contraction $C$ suggests a unitary dilation of the contractive stochastic cocycle $V_{t}$ in the quantum-mechanical sense. It should be given by a causal unitary cocycle $U_{t}$ in a Hilbert tensor product $\mathcal{H} \otimes \mathcal{F}_{\bullet}$ with respect to a free evolution unitary group $T_{t}$ in the additional space $\mathcal{F}_{\bullet}$ of an external quantum field, such that

$$
F_{t}^{*}(\omega) U_{t} F_{0} \chi=V_{t}(\omega) \chi(\omega), \quad \forall t \in R_{+}, \quad \omega \in \Omega .
$$

Here $\left(F_{t}\right)_{t>0}$ are isometries $\mathcal{H} \otimes L^{2}\left(\Omega, P^{\lambda}\right) \longrightarrow \mathcal{H} \otimes \mathcal{F} \bullet$ given as $F_{t}\left(\eta \otimes \varepsilon^{g}\right)=$ $\eta \otimes \varphi_{t}^{g}$ by a correspondence $\varepsilon^{g} \mapsto \varphi_{t}^{g}$ of the exponential test functions (3.9) of the Hilbert subspaces $L^{2}\left(\Omega, P^{\lambda}\right)$ and their representations $\varphi_{t}^{g} \in \mathcal{F}_{\bullet}$ such that

$$
\left\|\varphi_{t}^{g}\right\|^{2}=\int\left|\varepsilon^{g}(\omega)\right|^{2} P^{\lambda}(\mathrm{d} \omega)=\left\|\varphi_{0}^{g}\right\|^{2}, \quad \forall t \in \mathbb{R}_{+} .
$$

As follows from [9], a good candidate for $\mathcal{F}_{\bullet}$ is the Bosonic Fock space over the tensor product $\mathcal{K} \otimes L^{2}\left(\mathbb{R}_{+}\right)$of two dimensional $\mathcal{K}=\mathbb{C}^{2}$ and the space of square-integrable functions on $\mathbb{R}_{+}$such that $\mathcal{F}_{\bullet}$ is the Hilbert product $\mathcal{F}_{0} \otimes \mathcal{F}_{1}$ of two copies of the Fock space $\mathcal{F}$ isometric to the probabilistic space $L^{2}\left(\Omega, P^{\lambda}\right)$ for the Poisson process on $\mathbb{R}_{+}$. Realizing $\mathcal{F}_{\bullet}$ as the space of square-integrable 
tensor-functions $\varphi(\tau) \in \mathcal{K}^{\otimes|\tau|}$ of the finite sequences $\tau=\left\{r_{1}, \ldots, r_{n}\right\} \subset \mathbb{R}_{+}$, $r_{1}<r_{2}<\ldots<r_{n}$, we shall define the isometries $F_{t}$ by the tilde transform (3.10) of $\varepsilon^{g} \in L^{2}\left(\Omega, P^{\lambda}\right)$ as

$$
F_{t}\left(\eta \otimes \varepsilon^{g}\right)(\tau)=\eta \otimes \widetilde{\varepsilon}^{g}\left(\tau_{t}\right) e^{\otimes\left|\tau_{t}\right|} \otimes \widetilde{\varepsilon}^{g}\left(\tau_{[t}\right) e_{0}^{\otimes\left|\tau_{[t}\right|},
$$

where $e, e_{0} \in \mathcal{K}$ are unital 2-vectors, and $\left|\tau_{t}\right|=\tau \cap[0, t), \tau_{[t}=\tau \cap[t, \infty)$. The adjoint transform $F_{t}^{*}: \mathcal{H} \otimes \mathcal{F}_{\bullet} \rightarrow \mathcal{H} \otimes L^{2}\left(\Omega, P^{\lambda}\right)$ can be written as $\chi_{t}(\omega)=$ $F_{t}^{*}(\omega)(\eta \otimes \varphi)$ in terms of $\eta \otimes\left(e^{\otimes|\tau|} \mid \varphi(\tau)\right)$, where $e^{\otimes|\tau|}=e^{\otimes\left|\tau_{t}\right|} \otimes e_{0}^{\otimes\left|\tau_{[t}\right|}$, as the stochastic multiple integral;

$$
\chi_{t}(\omega)=\eta \otimes \int \lambda^{-|\tau| / 2}\left(e^{\otimes|\tau|} \mid \varphi(\tau)\right) \mathrm{d} m_{\tau} .
$$

Here we used the canonical decomposition $\mathcal{F}_{\bullet}=\mathcal{F}_{\bullet t} \otimes \mathcal{F}_{\bullet}[t$ to the Fock spaces over the orthogonal subspaces of square-integrable vector functions $f^{\bullet}=\left(f^{k}\right)$ on $[0, t)$ and $[t, \infty)$, respectively. The tensor multipliers $\mathcal{F}_{\bullet t}$ are increasingly embedded into $\mathcal{F}_{\bullet}$ as $\mathcal{F}_{\bullet t} \subset \mathcal{F}_{\bullet s} \forall s>t$ by $\mathcal{F}_{\bullet t} \ni \varphi_{t} \mapsto \varphi_{t} \otimes \varphi_{0}^{t}$, where $\varphi_{0}^{t}(\tau)=$ $\delta_{0}^{\left|\tau_{[t}\right|}$ is the vacuum normalized function of the space $\mathcal{F}_{[t}$.

The Hilbert space $\mathcal{H}_{t}=L_{\mathcal{H}}^{2}\left(\Omega, P_{t}^{\lambda}\right)$ of $\mathcal{H}$-valued stochastic causal functions $\chi_{t}(\omega)$ with the finite covariance

$$
\left\|\chi_{t}\right\|^{2}=\int\left\|\chi_{t}(\omega)\right\|^{2} P_{t}^{\lambda}(\mathrm{d} \omega)=\left\|\tilde{\chi}_{t}\right\|^{2}<\infty
$$

is causally represented by the initial isometry $F_{0}$ in the space $\mathcal{H} \otimes \mathcal{F}_{\bullet}$ and thus in $\mathcal{H} \otimes \mathcal{F} \bullet$ by the tilde transform $F_{0} \chi_{t}=\left(I \otimes e_{0}^{\otimes}\right) \tilde{\chi}_{t}$, where $e_{0}^{\otimes}$ is the embedding of $\mathcal{F}_{t}$ into $\mathcal{F}_{\bullet t}$,

$$
\left(I \otimes e_{0}^{\otimes}\right)\left(\eta \otimes \varphi_{t}\right)=\eta \otimes \varphi_{t} e_{0}^{\otimes}, \quad \varphi_{t} e_{0}^{\otimes}(\tau)=\varphi_{t}(\tau) e_{0}^{\otimes|\tau|},
$$

given by the tensor powers of the unit vector $e_{0} \in \mathbb{C}^{2}$. This representation is an isometry,

$$
\left\|F_{0} \chi_{t}\right\|^{2}=\left\|\left(I \otimes e_{0}^{\otimes}\right) \widetilde{\chi}_{t}\right\|^{2}=\left\|\chi_{t}\right\|^{2}
$$

due to the unitarity of the tilde transform $\chi \mapsto \widetilde{\chi}$. The adjoint co-isometry $F_{0}^{*}: \mathcal{H} \otimes \mathcal{F}_{\bullet} \rightarrow \mathcal{H} \otimes \mathcal{F}$ maps the localized kernels $\psi_{t} \in \mathcal{H} \otimes \mathcal{F}_{\bullet t}$ to the stochastic causal functions $\chi_{t}(\omega)=F_{0}^{*}(\omega) \psi_{t}$ given as the stochastic multiple integrals (3.4) with the kernels $\widetilde{\chi}_{t}=\left(I \otimes e_{0}^{\otimes}\right)^{*} \psi_{t}$ :

$$
\tilde{\chi}_{t}\left(r_{1}, \ldots, r_{n}\right)=\left(I \otimes e_{0}^{\otimes n}\right)^{*} \psi_{t}\left(r_{1}, \ldots, r_{n}\right) .
$$

Now we can describe the Markov quantum stochastic model of the unitary dilation (4.2), which has been found in 9] for the general CP flows $\phi_{t}^{g}$ over the algebra $\mathcal{B}(\mathcal{H})$ of all bounded operators in $\mathcal{H}$.

Let us assume that the contraction $C$ is dilated as in (4.1) to a unitary operator $S$ in $\mathcal{H} \otimes \mathcal{K}$, say, of the form (4.2) and take $e_{0}=\left(\delta_{0}^{k}\right), e=\left(\delta_{1}^{k}\right) \equiv e_{1}$. 
We shall define the unitary evolution $U_{t}$ in $\mathcal{H} \otimes \mathcal{F}_{\bullet}$ by the quantum stochastic differential equation (QSDE) in the sense of [18] as

$$
\mathrm{d} U_{t}+\mathrm{i} H U_{t} \mathrm{~d} t=\left(S_{k}^{i}-I \delta_{k}^{i}\right) U_{t} \mathrm{~d} N_{i}^{k}(t), \quad U_{0}=I \otimes \widehat{1},
$$

where $N_{k}^{i}(t)$ is the quantum stochastic Poisson matrix process of intensity $\lambda$ given by the canonical integrators in $\mathcal{F}$ as

$$
N_{k}^{i}(t)=\Lambda_{k}^{i}(t)+\sqrt{\lambda}\left(\Lambda_{-}^{i}(t) \delta_{k}^{0}+\delta_{0}^{i} \Lambda_{k}^{+}(t)\right)+\lambda \delta_{0}^{i} \delta_{k}^{0} t \widehat{1} .
$$

In the eigenrepresentation of the number process $N=N_{0}^{0}+N_{1}^{1}$ of total quantum number, the unitary solution to (4.1) can be written similarly to (2.4) as

$$
U_{t}(\omega)_{k_{1} \ldots k_{n}}^{i_{1} \ldots i_{n}}=S_{k_{n}}^{i_{n}}\left(t-t_{n}\right) \cdot \ldots \cdot S_{k_{1}}^{i_{1}}\left(t_{2}-t_{1}\right) \mathrm{e}^{-\mathrm{i} H t_{1}},
$$

where $S_{k}^{i}(t)=\mathrm{e}^{-\mathrm{i} H t} S_{k}^{i}, n=n_{t}(\omega)$, and $\omega=\left\{t_{1}, t_{2}, \ldots\right\}$ are the counting points for the total number process $N$ up to $t$ with the finite numbers $n_{t}(\omega)=$ $|\omega \cap[0, t)|$. We shall also define the isometries $F_{t}$ as $\left(I \otimes J_{t}\right) F_{0}$, where $J_{t}$ is a partial isometry given by the solution to QSDE

$$
\mathrm{d} J_{t}=\left(e e_{0}^{*}-\delta\right)_{k}^{i} J_{t} \mathrm{~d} N_{i}^{k}(t), \quad J_{0}=\widehat{1},
$$

where $e=e_{1}$ if the unitary matrix $S$ is taken in the form (4.3), (4.4). The equation (4.16) has the explicit solution

$$
J_{t}(\omega)=\left(e e_{0}^{*}\right)^{\otimes\left|\omega_{t}\right|} \otimes I^{\otimes\left|\omega_{[t}\right|},
$$

where $\omega_{t} \cup \omega_{[t}$ has a finite $\omega_{t}=\omega \cap[0, t)$. Note that the family of orthoprojectors $I_{t}=J_{t} J_{t}^{*}$, that is

$$
I_{t}(\omega)=\left(e e^{*}\right)^{\otimes\left|\omega_{t}\right|} \otimes I^{\otimes\left|\omega_{[t}\right|}
$$

satisfying

$$
\mathrm{d} I_{t}=\left(e e^{*}-\delta\right)_{k}^{i} I_{t} \mathrm{~d} N_{i}^{k}(t), \quad I_{0}=I \otimes \widehat{1},
$$

is decreasing, $I_{t} \leq I_{r}, \forall t \geq r \in \mathbb{R}_{+}$, describing the survival events for the detection of an unstable quantum particle by the time $t \in \mathbb{R}_{+}$. Obviously, $U_{t}$ dilates the stochastic evolution as $V_{t}(\omega)=U_{t}(\omega)_{0}^{1} \ldots 1$ coincides with (2.4) if $C=S_{0}^{1}$. Hence, the unitary evolution $U_{t} \psi_{0}$ of the initial state $\psi_{0}=F_{0} \chi$ with any $\chi \in \mathcal{H} \otimes L^{2}\left(\Omega, P^{\lambda}\right)$ defines the amplitude $\psi_{t}=\left(I \otimes J_{t}^{*}\right) U_{t} \psi_{0}$ normalized to the averaged survival probability (2.6)

$$
\left\|\psi_{t}\right\|^{2}=\left\langle U_{t} \psi_{0} \mid\left(I \otimes I_{t}\right) U_{t} \psi_{0}\right\rangle=\left\|F_{t}^{*} U_{t} F_{0} \chi\right\|^{2}=\left\|V_{t} \chi\right\|^{2}=q^{\lambda}(t) .
$$

Here we used the adaptedness of the solution $U_{t}$ in the sense

$$
U_{t}\left(\eta \otimes e_{0}^{\otimes} \varepsilon^{g}\right)(\tau)=U_{t}\left(\eta \otimes e_{0}^{\otimes} \varepsilon^{g}\left(\tau_{t}\right) \otimes e_{0}^{\otimes\left|\tau_{[t}\right|} \varepsilon^{g}\left(\tau_{[t}\right),\right.
$$

due to which $\left(I \otimes J_{t}^{*}\right) U_{t} F_{0}=F_{t}^{*} U_{t} F_{0}$. 
Let us also prove the dilation formula (4.5) using the generating functional method described in the Sec. 3, and find the limit of the unitary evolution as $\lambda \rightarrow \infty$ and $C=I-\lambda^{-1} R$. To do so it is more convenient to use another dilation, given by $e=e_{0}$ and the equation (4.13) with non-Hermitian unitary block matrix

$$
S=\left(\begin{array}{cc}
C & \left(I-C C^{*}\right)^{1 / 2} \\
-\left(I-C^{*} C\right)^{1 / 2} & C^{*}
\end{array}\right), \quad e=\left(\begin{array}{l}
1 \\
0
\end{array}\right)=e_{0} .
$$

We should find an equation for the coherent matrix elements

$$
U_{t}\left(\bar{g}^{\bullet}, f^{\bullet}\right)=\left(g^{\otimes} \mid U_{t} f^{\otimes}\right) / \exp \left\{\int_{0}^{\infty}\left(g^{\bullet}(r) \mid f^{\bullet}(r)\right) \mathrm{d} r\right\}
$$

and compare it with the equation (3.28) for $\breve{V}_{t}(\bar{g} \dot{+} f)=V_{t}(\bar{g}, f)$, where

$$
\begin{aligned}
V_{t}(\bar{g}, f) & =\int \overline{\varepsilon^{g}}(\omega) V_{t}(\omega) \varepsilon^{f}(\omega) P^{\lambda}(\mathrm{d} \omega) / \exp \left\{\int_{0}^{\infty} \bar{g}(r) f(r) \mathrm{d} r\right\} \\
& =\int \overline{\varepsilon_{t}^{g}}(\omega) V_{t}(\omega) \bar{\varepsilon}_{t}^{f}(\omega) P_{t}^{\lambda}(\mathrm{d} \omega) / \exp \left\{\int_{0}^{\infty} \bar{g}(r) f(r) \mathrm{d} r\right\} \\
& =\breve{V}_{t}(\bar{g} \dot{+} f) .
\end{aligned}
$$

Here we used the multiplication formula (3.7) and the independence of $\varepsilon_{t}^{\bar{g}+f} V_{t}$ and $\varepsilon_{[t}^{\bar{g} \dot{+} f}$, where $\varepsilon_{[t}^{f}=\varepsilon^{f_{[t}}\left(f_{[t}(r)=f(r)\right.$ if $r \geq t, f_{[t}(r)=0$ if $\left.r<t\right)$.

The equation for $U_{t}\left(\bar{g}^{\bullet}, f^{\bullet}\right)$ can be obtained by the substitution of the independent increments $\mathrm{d} N_{i}^{k}(t)$ for the number process (4.14) in (4.13) by their coherent matrix elements

$$
\left(f^{k}(t) g_{i}(t)+\lambda^{1 / 2}\left(\delta_{0}^{k} g_{i}(t)+f^{k}(t) \delta_{i}^{0}\right)+\lambda \delta_{0}^{k} \delta_{i}^{0}\right) \mathrm{d} t \widehat{1},
$$

where $g_{i}=\bar{g}^{i}$. Thus we obtain the ordinary differential equation

$$
\begin{aligned}
& \frac{\mathrm{d}}{\mathrm{d} t} U_{t}\left(\bar{g}^{\bullet}, f^{\bullet}\right)+\mathrm{i} H U_{t}\left(\bar{g}^{\bullet}, f^{\bullet}\right)= \\
& {\left[g_{i}(t)(S-I \delta)_{k}^{i} f^{k}(t)+\lambda^{1 / 2}\left(g_{i}(t)(S-I \delta)_{0}^{i}+(S-I \delta)_{k}^{0} f^{k}(t)\right)+\left(S_{0}^{0}-I\right) \lambda\right] U_{t}\left(\bar{g}^{\bullet}, f^{\bullet}\right) .}
\end{aligned}
$$

If $g_{i}=\delta_{i}^{0} \bar{g}, f^{k}=\delta_{0}^{k} f$ corresponding to the embeddings $g^{\bullet}=g e_{0}, f^{\bullet}=f e_{0}$, this equation indeed coincides with the equation (3.28);

$$
\begin{aligned}
& \frac{\mathrm{d}}{\mathrm{d} t} V_{t}(\bar{g}, f)+\mathrm{i} H V_{t}(\bar{g}, f) \\
& \quad=\left[\bar{g}(t)(C-I) f(t)+\lambda^{1 / 2}(\bar{g}(t)(C-I)+(C-I) f(t))+(C-I) \lambda\right] V_{t}(\bar{g}, f) \\
& \quad=(C-I)\left(\lambda^{-1 / 2}(g \dot{+} f)(t)+1\right) \lambda V_{t}(\bar{g}, f) .
\end{aligned}
$$

Here $V_{t}(\bar{g}, f)=U_{t}\left(\bar{g} \delta_{0}^{\bullet}, f \delta_{0}^{\bullet}\right)$ as

$$
\left\langle\xi \mid V_{t}(\bar{g}, f) \eta\right\rangle=\left\langle F_{0}\left(\xi \otimes \varepsilon^{g}\right) \mid U_{t} F_{0}\left(\eta \otimes \varepsilon^{f}\right)\right\rangle \exp \left\{-\int_{0}^{\infty} \bar{g}(r) f(r) \mathrm{d} r\right\}=\left\langle\xi \mid U_{t}\left(\bar{g} e_{0}, f e_{0}\right) \eta\right\rangle
$$


in the case $F_{t}=F_{0}$ corresponding to $e=e_{0}$.

Now, substituting $\mathrm{C}$ by $I-\lambda^{-1} R$ in (4.22) and taking into account that

$S=\left(\begin{array}{ll}I & 0 \\ 0 & I\end{array}\right)+\lambda^{-1 / 2}\left(\begin{array}{cc}0 & \left(R+R^{*}\right)^{1 / 2} \\ -\left(R+R^{*}\right)^{1 / 2} & 0\end{array}\right)-\lambda^{-1}\left(\begin{array}{cc}R & 0 \\ 0 & R^{*}\end{array}\right)+O\left(\lambda^{-3 / 2}\right)$,

let us find the limiting equation (4.24) as $\lambda \rightarrow \infty$. The right hand side of (4.24) up to the term of the order $\lambda^{-1 / 2}$ is written then as

$$
\left(O\left(\lambda^{-1 / 2}\right)+\left(R+R^{*}\right)^{1 / 2} f^{1}(t)-g_{1}(t)\left(R+R^{*}\right)^{1 / 2}-R\right) U_{t}\left(\bar{g}^{\bullet}, f^{\bullet}\right),
$$

giving to the equation

$$
\frac{\mathrm{d}}{\mathrm{d} t} U_{t}^{0}\left(\bar{g}^{\bullet}, f^{\bullet}\right)+(R+\mathrm{i} H) U_{t}^{0}\left(\bar{g}^{\bullet}, f^{\bullet}\right)=\left(R+R^{*}\right)^{1 / 2} U_{t}^{0}\left(\bar{g}^{\bullet}, f^{\bullet}\right)\left(f^{1}(t)-\bar{g}^{1}(t)\right)
$$

for the limiting $U_{t}^{0}\left(\bar{g}^{\bullet}, f^{\bullet}\right)=\lim _{\lambda \rightarrow \infty} U_{t}\left(\bar{g}^{\bullet}, f^{\bullet}\right)$. Equation (4.26) corresponds to the diffusion QSDE

$$
\mathrm{d} U_{t}^{0}+(R+\mathrm{i} H) U_{t}^{0} \mathrm{~d} t=\left(R+R^{*}\right)^{1 / 2} U_{t}^{0}\left(\mathrm{~d} \Lambda_{-}^{1}-\mathrm{d} \Lambda_{1}^{+}\right), \quad U_{0}^{0}=I \otimes \widehat{1},
$$

for the limiting unitary Markovian evolution, dilating the limiting nonstochastic contractive evolution (2.21) for $V_{t}^{0}=\lim _{\lambda \rightarrow \infty} V_{t}$. It is driven by the momentum process

$$
P_{t}=\mathrm{i}\left(\Lambda_{1}^{+}-\Lambda_{-}^{1}\right)(t)=\widehat{w}_{t}
$$

which is the quantum stochastic representation of the standard Wiener process $w_{t}$ in the Fock space $\mathcal{F}_{1}$, the copy of the original Fock space $\mathcal{F}_{0}$ for the representation $N_{0}^{0}=\widehat{n}_{t}$ of the Poisson process $n_{t}(\omega)$.

Thus, the quantum stochastic unitary evolution for the unstable particle dilating the process of weakly random contractions $C=I-\lambda^{-1} R$ due to frequent detection of the particle at random times with the rate $\lambda \rightarrow \infty$ becomes classically stochastic. The time-evolution of its pure state is described by the Itô-Schrödinger equation

$$
\mathrm{d} \psi_{t}^{0}+(R+\mathrm{i} H) \psi_{t}^{0} \mathrm{~d} t=\mathrm{i}\left(R+R^{*}\right)^{1 / 2} \psi_{t}^{0} \mathrm{~d} w_{t}
$$

for $\psi_{t}^{0}\left(\omega_{1}\right)=U_{t}^{0}\left(\omega_{1}\right) \eta$. Here $\omega_{1}$ is an elementary event of the standard Wiener probability space $\left(\Omega_{1}, P_{1}\right), H=H^{*}$ is a selfadjoint operator, and $R+R^{*} \geq 0$ is the rate operator for the contraction semigroup $\mathrm{e}^{-K t}, K=R+\mathrm{i} H$.

Eq. (4.29) provides a new dynamical formulation of the quantum Zeno effect 10, 11. The limiting dynamics becomes reversible (invertible) as the reductions of the increasing rate and decreasing amplitude can be compensated by field fluctuations given by the momentum process. Let us stress that the large number limit (4.29) of the unitary dilation of the contractive stochastic dynamics remains stochastic. To our knowledge, the stochastic dynamics has not been obtained so far, in a similar context.

Acknowledgement This work supported in part by The State Committee for Scientific Research, under the project no. 2P03B 129 11. Dedicated to R.S. Ingarden on the occasion of his $80^{\text {th }}$ birthday 


\section{References}

[1] R. Zwanzig, Physica 30, 1109 (1964).

[2] K. Sinha, Helv. Phys. Acta 45, 619 (1972).

[3] L. P. Horwitz, J. A. La Vita, and J. P. Marchand, J. Math. Phys. 12, 2537 (1971).

[4] D. N. Williams, Commun. Math. Phys. 21, 314 (1971).

[5] B. Sz.-Nagy, C. Foias, Harmonic analysis of operators on Hilbert space, North Holland, Amsterdam 1970.

[6] M. Ozawa, J. Math. Phys. 25, 79 (1984).

[7] E. B. Davies, Quantum Theory of Open Systems, Academic Press, London 1976.

[8] L. Fonda, G. C. Ghirardi, A. Rimini, Nuovo Cimento, 18B, 1 (1973).

[9] V.P. Belavkin, Commun. Math. Phys. 184, 553 (1997).

[10] Ch. N. Friedman, Indiana Univ. Math. J. 21, 1001 (1972).

[11] B. Misra and E. C. G. Sudarshan, J. Math. Phys. 18, 756 (1977).

[12] V.P. Belavkin, in Information Complexity and Control in Quantum Physics, edited by A. Blaquiere, S. Diner, and G. Lochak, Springer, Berlin, 1987, p. 311, V.P. Belavkin, in Modeling and Control of Systems in Engineering, Quantum Mechanics, Economics and Biosciences, edited by A. Blaquiere, Springer, Berlin, 1988, p. 245, V. P. Belavkin, in Stochastic Methods in Mathematics and Physics, edited by R. Gielerak and W. Karwowski, World Scientific, Singapore 1989, p. 310,

[13] A. Barchielli, V. P. Belavkin, J. Phys. A: Math. Gen. 24, 1495 (1991).

[14] V. P. Belavkin, Commun. Math. Phys. 146, 611 (1992).

[15] P. Staszewski, Quantum Mechanics of Continuously Observed Systems, N. Copernicus University Press, Torun, 1993.

[16] V. P. Belavkin, Foundations of Physics 24, 685 (1994).

[17] S. Albeverio, V. N. Kolokol'tsov, and O. G. Smolyanov, Rev. Math. Phys. 9, 907 (1997).

[18] R. L. Hudson and K. R. Parthasarathy, Commun. Math. Phys. 93, 301 (1984).

[19] D. Goswami, J. M. Lindsay, K. B. Sinha, and S. J. Wills, Dilation of Markovian Cocycles on a von Neumann Algebra, The University of Nottingham, Preprint (1999). 